\title{
The NIR arm of SHARK: System for coronagraphy with High-order Adaptive optics from $\mathbf{R}$ to $\mathbf{K}$ bands
}

\author{
Jacopo Farinato', Carlo Baffa ${ }^{2}$, Andrea Baruffolo', Maria Bergomi ', Luca Carbonaro², \\ Alexis Carlotti ${ }^{3}$, Mauro Centrone ${ }^{4}$, Johanan Codona ${ }^{5}$, Marco Dima', \\ Simone Esposito', Daniela Fantinel ', Giancarlo Farisato', Wolfgang Gaessler ${ }^{6}$, \\ Emanuele Giallongo ${ }^{4}$, Davide Greggio', Philip Hinz ${ }^{5}$, Franco Lisi ${ }^{2}$, Demetrio Magrin', \\ Luca Marafatto', Fernando Pedichini ${ }^{4}$, Enrico Pinna ${ }^{2}$, Alfio Puglisi ${ }^{2}$, \\ Roberto Ragazzoni ', Bernardo Salasnich', Marco Stangalini ${ }^{4}$, Christophe Verinaud ${ }^{3}$ \\ and Valentina Viotto' \\ ${ }^{1}$ INAF Padova, Vicolo dell'osservatorio 5, 35122 Padova, Italy \\ e-mail: jacopo.farinato@oapd.inaf.it \\ ${ }^{2}$ INAF Arcetri, Largo Enrico Fermi, 5, 50125 Firenze, Italy \\ ${ }^{3}$ Institut de Planétologie et d'Astrophysique de Grenoble, 414, Rue de la Piscine, Domaine Universitaire, 38400 St-Martin \\ d'Hères, France \\ ${ }^{4}$ INAF Roma, Via di Frascati 33, 00040 Monte Porzio Catone, Italy \\ ${ }^{5}$ Department of Astronomy/Steward Observatory, University of Arizona, 933 North Cherry Avenue, Tucson, AZ, USA \\ ${ }^{6}$ Max Planck Institute for Astronomy, Königstuhl 17, 69117 Heidelberg, Germany
}

\begin{abstract}
SHARK is a proposal aimed at investigating the technical feasibility and the scientific capabilities of high-contrast cameras to be implemented at the Large Binocular Telescope (LBT). SHARK foresees two separated channels: near-infrared (NIR) channel and visible, both providing imaging and coronagraphic modes. We describe here the SHARK instrument concept, with particular emphasis on the NIR channel at the level of a conceptual study, performed in the framework of the call for proposals for new LBT instruments. The search for giant extra-Solar planets is the main science case, as we will outline in the paper. Received 9 May 2014, accepted 7 September 2014, first published online 10 October 2014
\end{abstract}

Key words: coronagraphy, extreme adaptive optics, large binocular telescope, planet finding.

\section{Introduction}

The Large Binocular Telescope (LBT), with its First Light Adaptive Optics (FLAO) systems, recently (first scientific light in May 2010, see Esposito et al. 2010, 2011, 2012; Close et al. 2012a) opened a new frontier for astronomical Adaptive Optics (AO) on 8-10 m class telescopes. The combination of the pyramid-based sensor (see Ragazzoni 1996; Ragazzoni \& Farinato 1999; Esposito \& Riccardi 2001; Ghedina et al. 2003; Costa et al. 2004; Vérinaud et al. 2005), together with the high dynamic and spatial resolution of the adaptive secondary mirror (ASM, see Salinari et al. 1994; Riccardi et al. 2010) provides performance never reached on this class of telescopes by previous natural or laser guide star systems. In particular, we refer to the extremely low residual wavefront error (below 100 $\mathrm{nm}$ RMS) routinely reached by FLAO systems working with bright guide stars. This amount of residual translates into the first high-contrast AO-corrected images in the NIR, with Strehl Ratios (SRs) greater than $90 \%$ in $\mathrm{K}$ and $\mathrm{H}$ bands and better than $60 \%$ in $\mathrm{J}$ (see Quiròs-Pacheco et al. 2011). Currently, the FLAO system is the only one able to deliver a contrast (without coronagraphic techniques) higher than $10^{4}$ in $\mathrm{H}$ band at a distance of $\sim 350$ mas (see Fig. 1) and even better in $\mathrm{K}$ band.
The high level of AO correction also delivers a good image correction at shorter wavelengths, reaching SR of $45 \%$ in $\mathrm{R}$ band.

From the seeing point of view, Mount Graham has shown a remarkably good behaviour (see Masciadri et al. 2010; Hagelin et al. 2011), with an average seeing of approximately $1^{\prime}$ achieved during the commissioning of FLAO, with several nights in which the seeing has been lower than $0.8^{\prime}$ and sometimes as good as $0.3^{\prime}$ in V-band.

The excellent Strehl performance opens up a variety of unexplored science cases (Rodigas et al. 2012; Esposito et al. 2013; Close et al. 2014), the most important of which being, of course, planet finding. The challenge of direct imaging planet detection is in the high contrast necessary between the star and the planet at very small separations. The contrast achieved in the NIR and the performance reached at visible wavelengths with FLAO at LBT are the starting point for proposing a new facility designed for high-contrast imaging.

SHARK will have two channels, each of them installed on a different telescope arm, namely the VISible channel (VIS channel in the following) and the Near InfraRed channel (NIR channel in the following), covering the region between $\mathrm{R}$ - and $\mathrm{K}$ bands.

The two channels of SHARK are simple and flexible instruments, essentially two cameras for direct imaging (both with a 

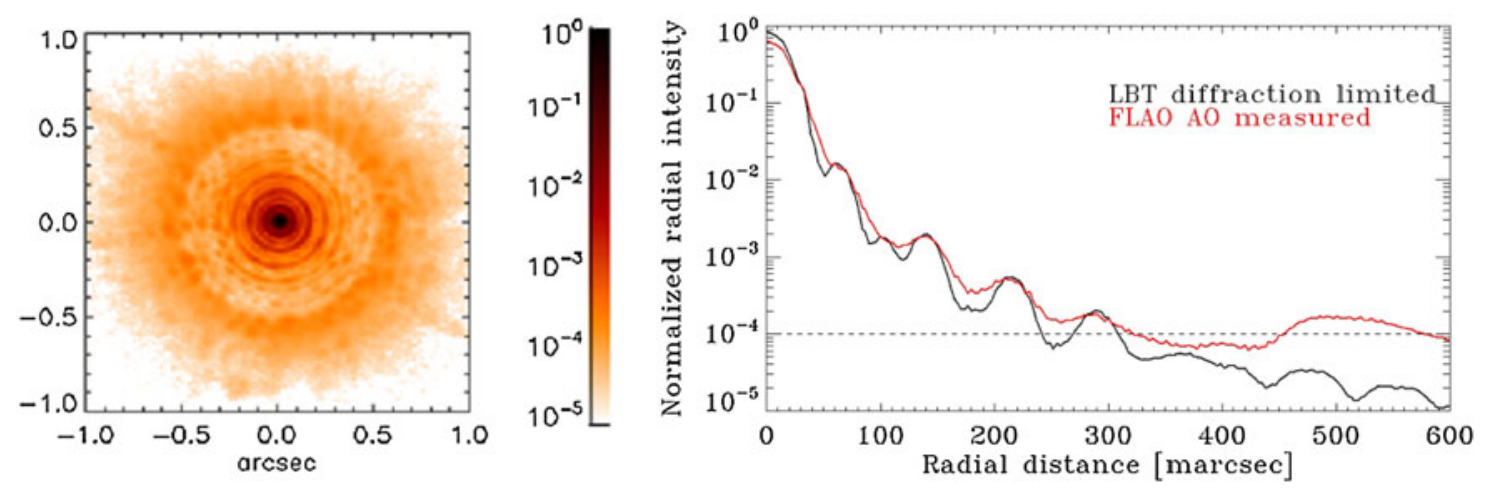

Fig. 1. (Left) High-order AO-corrected PSF using a bright star $\left(\mathrm{MR}=6.5\right.$ ) under median seeing conditions (seeing $\left.\sim 0.7^{\prime} \pm 0.1^{\prime}\right)$. (Right) Comparison between the diffraction-limited PSF (black full curve) and the AO-corrected PSF profiles (red full curve). Profiles are normalized to the diffraction-limited peak.

field of view (FoV) of the order of $20^{\prime} \times 20^{\prime}$ ) but equipped with coronagraphic capabilities. In both channels, we foresee a spectroscopic option:

- In the VIS channel, the baseline design has a fibre-fed Integral Field Unit (IFU), in a $2^{\prime}$ region, with spaxel (which is the volumetric pixel, the pixel in three-dimensional (3D) space) in the range 40-80 mas and spectral resolution (normally indicated with $R=\lambda / \Delta \lambda$ ) in the range 100-5000.

- In the NIR channel, there may be a spectroscopic option through a long slit coupled to a Grism, with a spectral resolution of the order of $R \sim 100$

In both channels, it is possible to insert polarimetric filters, together with several broad- and narrow-band filters.

We stress that the VIS channel of SHARK is unique (in every observing mode, i.e. imaging, coronagraphy and spectroscopy) in the current LBT instruments scenario, whereas the NIR channel is complementary (above all in its coronagraphic mode) to other existing observing facilities, such as LUCI ('Lbt nir spectrograph Utility with Camera and Integral-field unit', see Mandel et al. (2007)) and LBTI ('LBT Interferometer', see Hinz et al. (2003)). In fact, the LUCI NIR camera is not optimized for very high contrast and is not equipped with a coronagraph (see Mandel et al. 2007), whereas the coronagraph of LBTI operates in L $-\mathrm{M}$ bands (see Wilson et al. 2008). We also emphasize that SHARK is proposed for the LBT second generation instrumentation but, in the proposed configuration, it may co-exist with the currently available instrumentation, being placed at the entrance of LBTI, sharing part of the wavelength domain.

The international situation concerning coronagraphy is very favourable for such an instrument in the LBT framework. In fact, Spectro-Polarimetric High-contrast Exoplanet RE-search instrument for the VLT (SPHERE; see Beuzit et al. (2010)), Gemini Planet Finder (GPI; see Macintosh et al. 2008; Thomas et al. 2011) and Magellan AO (MagAO; see Close et al. 2012b; Males et al. 2013), which are currently under installation or commissioning at VLT in Paranal, at Gemini South and at the Magellan telescope respectively, will all operate in the Southern Hemisphere.

The two main coronagraphic instruments operating in the Northern Hemisphere, the Subaru Coronagraphic Extreme
Adaptive Optics (SCExAO; see Guyon et al. 2011) and Palm-3000 (Palomar AO; see Dekany et al. 2013a, b), even though very interesting and obtaining preliminary, on-sky results (see Currie et al. 2013; Dekany et al. 2013a, b), are far from obtaining the Extreme AO (XAO) performance of LBT, especially for faint targets.

We recall that Kepler is creating the biggest ever catalogue of exoplanet candidates, which lie in the small region (about $10^{\circ}$ in diameter), centred at a declination of $44^{\circ} 30^{\prime}$. This implies that the maximum elevation reached by this region will be $21^{\circ}$ (air mass $=2.8$ ) from VLT, $15^{\circ}$ (air mass $=3.9$ ) from Gemini South and $77^{\circ}$ (air mass $=1.02$ ) from LBT. For this reason, LBT will be more suitable for observing the Kepler region with an XAO planet finder. The superb science cases, the international scenario, the expertise of a few LBT partners and the fantastic performance of the LBT XAO are the basis for this proposal.

\section{A SHARK hunting for planets}

The detection of extra Solar planets is one of the most exciting scientific pursuits of modern Astronomy. Owing to the very demanding resolution and contrast required at the scientific images, it is a very challenging task to achieve with direct imaging. In fact, previous discoveries in this field have been possible mostly using non-direct imaging techniques, such as 'transits' and 'radial velocity' measurements, which revealed a large number of planets and planetary systems very different from the Solar one, improving our knowledge on the planets formation and evolution issue. The transit technique consists essentially in detecting, through very accurate photometry, the changes in the star flux due to the planet transit on the hosting star. The radial velocity technique is detecting instead the Doppler spectral line shift due to the periodic perturbation introduced by the rotating planet on the star position with respect to the observer.

Such techniques have a few intrinsic limitations:

- Both have a limited distance at which the planet detection is possible (a few astronomical units), radial velocity because the perturbation caused by the planet on the velocity is decreasing with the distance, transit because is less probable to 
have a transit along the line of sight if the planet is far away from the star.

- Both have a limited sample of old and chromo spherically quiet stars to observe, otherwise the fluctuations on the flux would make the detection impossible.

- The radial velocity technique is further limited to observe slowly rotating star, otherwise the intrinsic rotation would smear the lines in the spectrum decreasing the planet detectability.

- Both have a limited capability of characterizing the discovered planet, the radial velocity giving only a lower limit to the planet mass and the transit giving only the planet radius. Only recently, a combination of XAO with suitable diffraction (coronagraphs) and speckle subtraction techniques (angular differential imaging-ADI, spectral differential imagingSDI and polarimetric differential imaging) made it possible to reach the correct regime for the detection of giant planets around young, nearby stars through 'direct imaging'. The latter gives the possibility to observe planetary systems around stars in a wide range of masses and distances (the only limit being the resolving power of the telescope) and to characterize photometrically and spectroscopically the detected planets. In addition, the usage of a coronagraph to enhance the contrast between the hosting star and the planet increase the detection capability of the instrument.

Since the performance of the AO system is better at longer wavelengths (due to the direct wavelength dependence of the Fried diameter), the natural application of Coronagraphy is in the IR domain, where the achieved SR is giving remarkable contrast, which, as already said, is necessary for faint companion detection.

The mechanism of formation of planetary systems, where the outer regions are among the most critical to understand, is of great interest for such kind of instruments. Current available data mainly concern planets at small separations. The search for planets at large separation is a common scientific target of the recent years; in this field, the most sensitive technique is high-contrast imaging of young stars. Close-in or old planets mainly shine through reflected light, but such planets are expected to be projected very close to the central star and are difficult to detect. Young stars should then be preferred because planet intrinsic luminosity mainly depends on gravitational contraction and rapidly declines while the planet ages. These objects are better observed in the NIR $(1-2.5 \mu \mathrm{m})$. Up to now, we know only two systems (HR8799 and $\beta$ Pic) with characteristics typical of planetary systems. Although both of them are extremely interesting objects, better statistics are clearly crucial to constraining the models and characterizing the newly discovered planets. Detection of multiple systems and, of orbital motion, allows a discussion of the dynamical properties of the system. In addition, there is evidence that the smallest (young) planets do not follow the same relation between colour and spectral type found for Brown Dwarfs, the $\mathrm{L}-\mathrm{T}$ transition possibly occurring at a lower temperature.

Given the characteristics of the LBT AO-systems with respect to those of both SPHERE and GPI, we expect targets 2-3 magnitudes fainter to be reachable, and this will allow the observation of many more nearby, small mass stars and Solar-type stars in star-forming regions (e.g. Taurus at $140 \mathrm{pc}$ ). The proposed instrument will allow the LBT to study the earliest evolution of giant planets and their link with the circumstellar discs, thus providing specific clues to the formation mechanism.

\section{SHARK at the telescope}

As already mentioned, the basic idea is to have one SHARK channel installed on each LBT arm. There is a preliminary agreement with the LBTI PI (Phil Hinz) in order to install SHARK at the entrance foci of LBTI, as shown in Fig. 2, using two deployable dichroics to feed the two SHARK channels. In this way, on the VIS side, the IR light is totally transmitted to LBTI, whereas on the NIR side, only wavelengths longer than K band would reach the LBTI focus. The location of the dichroics is just before the entrance window of LBTI, the latter transmitting the IR light to the interferometric focus and reflecting the VIS light to the Pyramid WFS. Such a dichroic, on the VIS channel, would pickup only a certain amount (to be decided) of the VIS light to be used by the scientific camera, leaving the rest of the light to go to the WFS. On the NIR channel, the dichroic is picking up only the $\mathrm{J}, \mathrm{H}$ and $\mathrm{K}$ bands, letting all the visible light and the wavelengths longer than K to go through (to the WFS and to LBTI, respectively).

With this setup, SHARK will provide possible simultaneous observations from R to K bands. Such a flexible configuration, with several combined binocular observing modes, reflects the request from the principal science cases, for which simultaneous observations in the VIS and the NIR domain are required.

In the following, after some considerations on the possible coronagraphic solutions, we describe the conceptual optomechanical study of the NIR and VIS channels.

\section{SHARK coronagraphy}

The limited volume available in the current location where SHARK might be installed, the need to keep the design of SHARK as simple and compact as possible, and the wish to minimize the number of optical elements in the design are the drivers for selecting a simple classical Lyot (see Lyot 1939) as the baseline coronagraph. We briefly recall that such a configuration has an occulting mask to be positioned on the focal plane to mask the star light, and a stop on the pupil plane to undersize the pupil to minimize diffractive effects caused by the occulting focal plane mask.

The classical Lyot Coronagraph works quite well with Inner

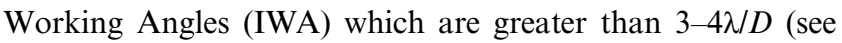
Guerri et al. 2009; Guerri et al. 2011), and thus does not allow one to go closer than $\sim 200$ mas from the star in $\mathrm{K}$ band ( $\sim 80$ mas in $\mathrm{R}$ band). Of course, there are science cases, which would benefit a lot from going as close as possible to the central object, and this is why we are carefully evaluating other coronagraphic techniques. To achieve this, we do have a design (see the following sections) where, both onto the focal 


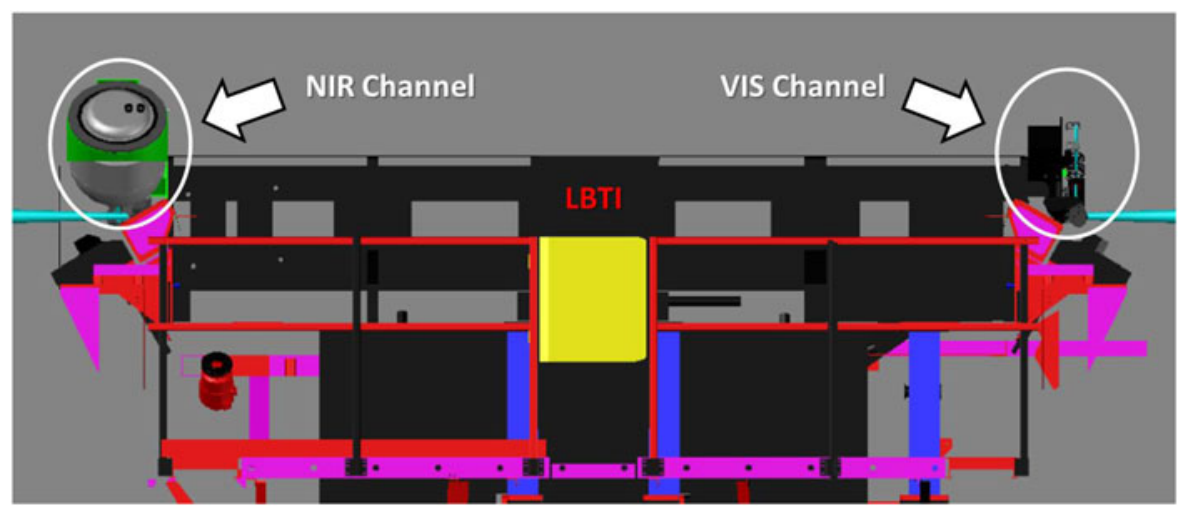

Fig. 2. A possible installation of the two SHARK channels at the LBTI entrance foci.

plane and onto the pupil plane, there are wheels that can accommodate up to eight masks. We emphasize that alternative coronagraphic techniques will not impact the instrument design, which is kept as the minimum level of complexity required for an imaging camera (above all in the NIR channel, where a re-imaging of the pupil is anyhow necessary to introduce a cold stop to minimize the thermal background). We will in fact consider only coronagraphic techniques that require an additional mask in the currently available focal plane or pupil plane, with the only impact being to fill one of the available positions in the corresponding filter wheel, as it will be clear in the section describing the opto-mechanical layout of the instrument.

The world of coronagraphic techniques is, in fact, rather wide, and there are several techniques differing significantly (see Martinez et al. 2008) concerning:

- The possible achievable IWA;

- The working wavelength (some working better in the visible, some better in the NIR, some being essentially monochromatic and thus suitable only with narrow bands filters);

- Their sensitivity to misalignment (vortex mask, for example, hat to be placed very precisely onto the focal plane);

- Their transmission (ranging from 10 to $90 \%$ )

Even discarding all the techniques that require a re-imaging of the pupil before the focal plane (apodized Lyot for example), which is not foreseen in SHARK, there are a few interesting options that might be implemented such as, for instance, Shaped Pupil coronagraph (see Carlotti et al. 2012), Phase Apodizing coronagraphy (see Codona \& Angel 2004) and Vortex coronagraph (see Mawet et al. 2005). These techniques work with an IWA that can be as small as $1-2 \lambda / D$ and with higher contrast than a simple Lyot coronagraph, and they are still compatible with the current design. We will carry out a comprehensive study of possible alternative techniques, with the goal of having a few choices matching as far as possible the requirements of the different science cases.

\section{The opto-mechanical concept}

As already shown in the section 'SHARK at the telescope', the two SHARK channels (VIS and NIR) will be installed one for each LBT arm. In this section, we describe the optomechanical layout of the two channels, starting with the NIR.

\section{NIR channel opto-mechanical layout}

The NIR channel of SHARK will provide both direct and coronagraphic imaging, over a FoV of about $20^{\prime} \times 20^{\prime}$ (almost $30^{\prime}$ on the diagonal). The current optical design is described in Fig. 3 left side, and is based on a classical two off-axis parabolas design, with the aim of maximizing the optical quality on the FoV (SR > 97.7\%) and minimizing the instrument dimensions. All the optics used inside the instrument is below $40 \mathrm{~mm}$ in diameter, demanding in terms of optical quality, but commercially available. The overall NIR channel can rotate on a mechanical bearing to compensate for field rotation.

Just before the input focal plane, there is a deployable atmospheric dispersion corrector (ADC), to correct for atmospheric dispersion. The optical quality of the system remains very good in the entire FoV, both in the term of encircled energy (larger than $80 \%$ for the $\mathrm{J}$ band diffraction limit point-spread function (PSF)) and in term of SR (always larger than 97.7\% with and without ADC), as one can see in Table 1. We achieve also, with this configuration, a good quality of the pupil image; in fact, the broadening in the image of a point of the pupil is $<10 \mu \mathrm{m}$, which roughly corresponds to $0.07 \%$ of the pupil's image diameter.

Continuing with the description, on the input focal plane, a filter wheel can select between different occulting masks.

On the pupil plane, a selector can switch between the Lyot mask and the 'empty' position (to provide direct NONCoronagraphic imaging on the detector). Six additional mask positions are available here, giving a total of eight.

A filter wheel positioned just after the pupil plane (seven positions available), making it possible to insert broad and narrow band filters.

Just after, another filter wheel (seven positions in total) allows the insertion of different neutral density filters, to permit observations of very bright targets. This wheel might possibly also accommodate a few polarized filters or additional science filters, for science cases for which only faint target observation is required.

The second off-axis parabola and a couple of folding mirrors direct the beam towards the HAWAII II detector, on which the spatial sampling is 2 pixels on the $\mathrm{J}$-band diffraction limit PSF, i.e. Nyquist sampled in $\mathbf{J}$. 

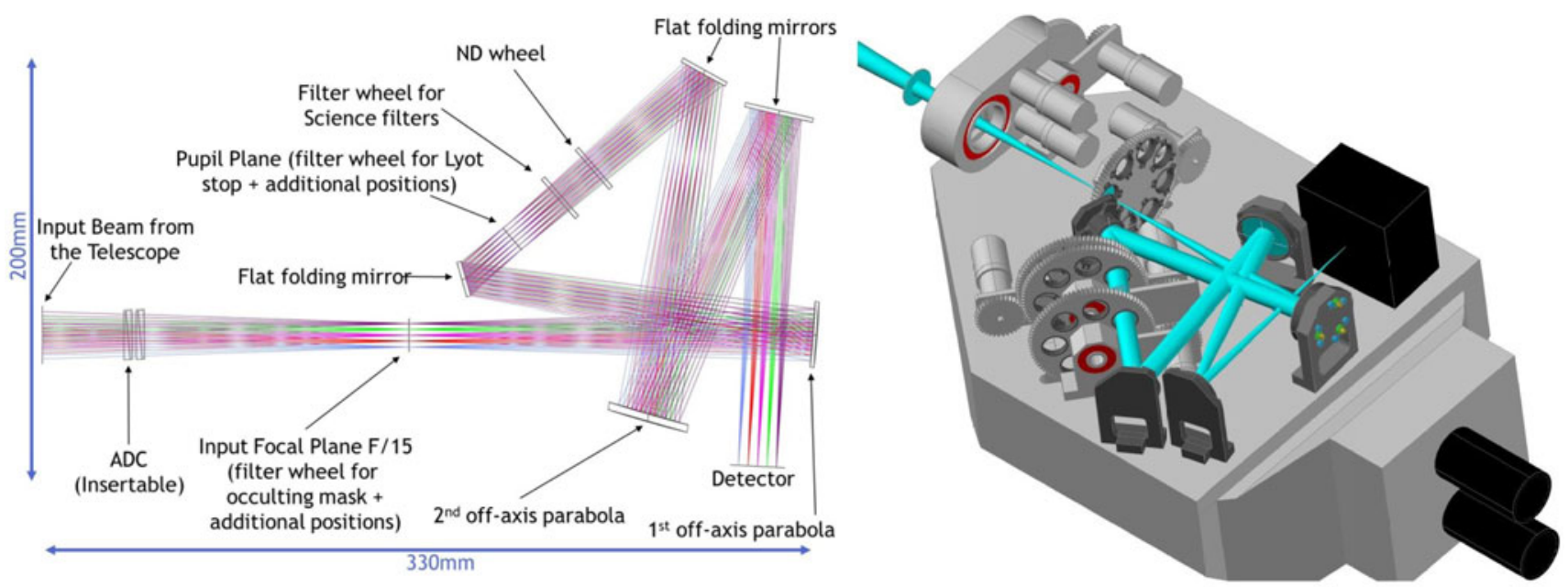

Fig. 3. The SHARK NIR channel optical design (left side) and opto-mechanical concept (right side).

Table 1. The main characteristics of the SHARK NIR channel

\begin{tabular}{ll}
\hline Observing modes & Imager/coronagraph \\
\hline Field of view (arcsec) & $19.2 \times 19.2$ \\
Wavelength range $(\mu \mathrm{m})$ & $1.1-2.4$ \\
Sampling (marcsec pix ${ }^{-1}$ ) & 19 \\
Encircled energy at 37 mas $(\mathrm{J}$ band & $>83 \%$ \\
$\quad$ diffraction limit PSF) & $>99.8 \%$ \\
Intrinsic optical quality $\left(0^{\circ}-25^{\circ}\right.$ & \\
$\quad$ Zenithal distance - No ADC) [SR] & $>97.8 \%$ \\
Intrinsic optical quality (30 & \\
$\quad$ Zenithal distance with ADC) [SR] & $>97.7 \%$ \\
Intrinsic optical quality (45 & Lyot (+TBD?) \\
$\quad$ Zenithal distance with ADC) [SR] & TBD (J, H, \\
Coronagraph type & K + Narrow Bands?) \\
Filters & TBD \\
Polarimetric filters & \\
\hline
\end{tabular}

The possibility of polarimetry (both in direct imaging and coronagraphy) using polarized filters inserted into the optical path is under study, as well as the possibility of low-resolution spectroscopy using a long slit and a grism inserted on the focal plane and collimated beam, respectively. At this stage, both solutions might be easily implemented (of course if requested by the science cases) by adding the appropriate optics to the current filter wheels. Figure 3 (right side) and Fig. 4 (left side) show 3D views of the NIR channel opto-mechanical study, which of course foresees dedicated cryogenic gears and stepper motors, initialized through dedicated limit switches.

The Cryogenic tank, shown in the left of Fig. 4, can contain, in this preliminary design, 20 litres of nitrogen, ensuring a duty cycle for the refill of more than $24 \mathrm{~h}$. The refilling valves have been oriented in such a way that the maximum angle with respect to the zenith position is $45^{\circ}$, independently from the telescope position, to avoid nitrogen spilling out.

The detector foreseen is a Hawaii $2 \mathrm{RG} \mathrm{HgCdTe} \mathrm{detector,} \mathrm{of}$ which we will use only one quadrant, meaning that we will use $1024 \times 102418 \mu \mathrm{m}$ pixels. The housing is custom-made, and the dimensions currently considered in the opto-mechanical design have been taken from similar systems already in use, and thus are an upper limit to the volume that the detector may occupy.

\section{VIS channel opto-mechanical layout}

We briefly describe also the VIS arm of SHARK, which has a layout very similar to that of the NIR channel, the main difference being the use of a moveable mirror to allow direct imaging (bypassing the coronagraphic path) of the whole FoV (about $20 \times 20 \mathrm{arcsec}$ ) on the camera detector. In this configuration (direct imaging), the light coming from the telescope passes only through the ADC (also removable) and through a flat mirror, as can be seen in Fig. 5 (where the flat mirror, called 'direct imaging selector mirror', is not inserted in the optical path).

When the first flat mirror is not inserted (as shown in Fig. 5), the coronagraphic optical layout is based on two off-axis parabolas giving a $5 \times 5$ arcsec corrected field of view (Strehl $>99 \%$ ). Two filter wheels, one in the focal plane and one in the pupil plane, allow for the selection of occulting masks and pupil stops. This configuration is suitable for the Lyot coronagraph, but can also make use of Vortex, shaped pupil coronagraphs and Phase masks. A third wheel after the pupil plane is available for science filters.

The field rotation may be provided by means of a direct camera rotation due to its low weight $(<1 \mathrm{~kg})$. This solution has the advantage of minimizing variation in the non-common path aberrations. The camera will move on a motorized focus adjustment, not shown in the image. We foresee to use a fast, lownoise sCMOS camera with a $2500 \times 2000$ pixels detector and a very small pixel size of $6.5 \mu \mathrm{m}$. This device can expose subfields of $256 \times 256$ pixels at about 1000 frame per seconds with a 1e-rms noise level allowing imaging of bright sources with high dynamics and freezing of the speckles.

Figure 5 shows an overall view of the compact $(450 \times 400 \mathrm{~mm})$ VIS channel, for which a spectroscopic mode (also not shown in the image) is also foreseen. This will use a fibre-fed IFU 

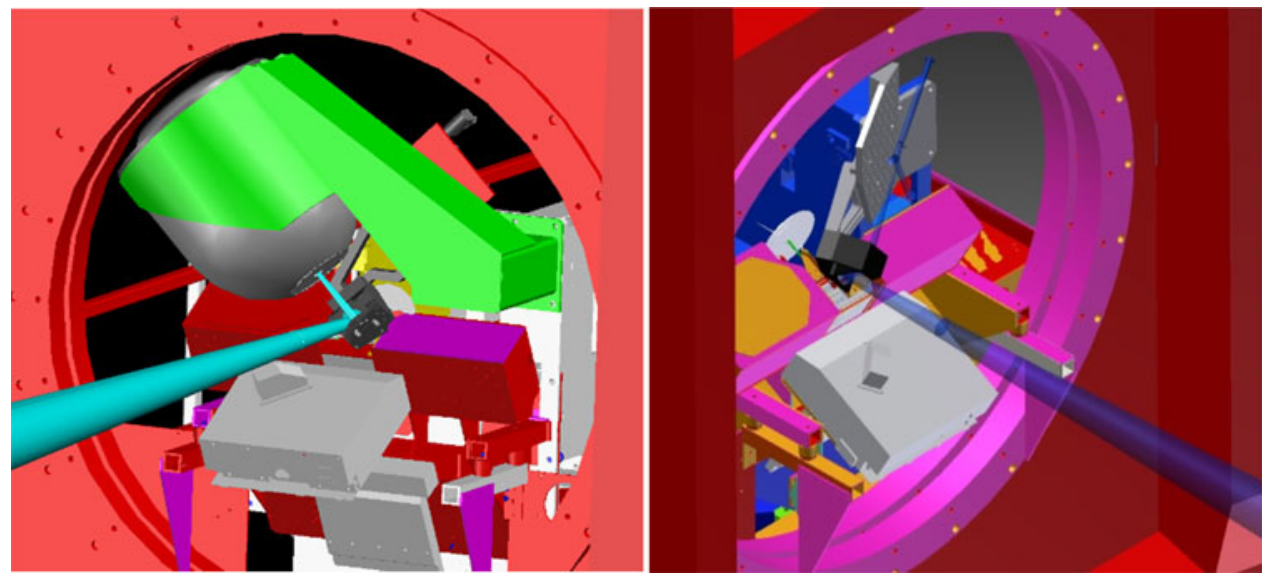

Fig. 4. SHARK (NIR channel on the left, VIS channel on the right) installed at the LBTI entrance focus.

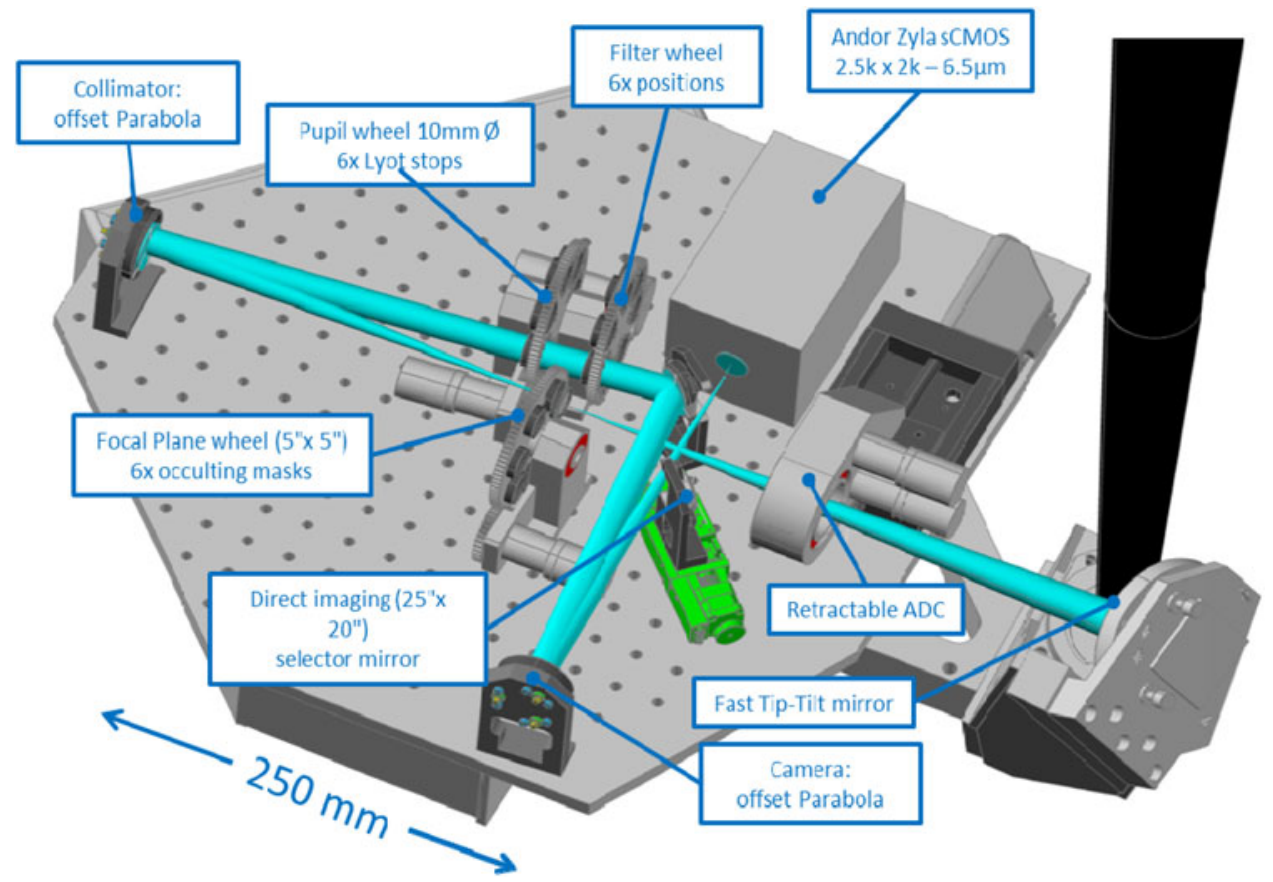

Fig. 5. CAD render of the assembled VIS channel, in green one can see the removable relay mirror used to exploit direct imaging, when inserted in the optical path. The pitch of the holes on the optical breadboard is $25 \mathrm{~mm}$.

mode, which will be accomplished by moving the camera away in the 'extra-focal' direction and inserting the optical fibre bundle onto the focal plane. The latter interfaces the VIS channel focal plane to an external slit spectroscope (located in a convenient location at a certain distance from the instrument) allowing multi-spectral images of a $2 \times 2$ arcsec subfield with spatial resolutions of about 40-80 mas. The spectral coverage is in the range $600-950 \mathrm{~nm}$, whereas the spectral resolution depends on the flux of the sources and final design of the spectroscope, with probably a couple of selectable spectral resolutions ranging from a few hundred to a few thousand. The VIS channel is installed on the side of the LBTI input port, as already shown in Fig. 2. In Fig. 4 (right), there is a more detailed view of the possible installation area on the LBTI side, where a deployable arm will allow the insertion of a dichroic, picking up a fraction of the order of $50 \%$ of the visible light folding it towards SHARK, leaving the rest of the light to reach the wave front sensor. All the IR light is untouched giving, in principle, the possibility of operating LBTI and the VIS channel of SHARK at the same time.

\section{Critical issues}

We have identified what we believe are the most important critical issues in the design and operation of SHARK, and we briefly describe the current plan to address them.

\section{Tracking the PSF movements}

Changes in the opto-mechanical setup during the exposure (due to flexures, thermal effects, etc.) can cause misalignments 

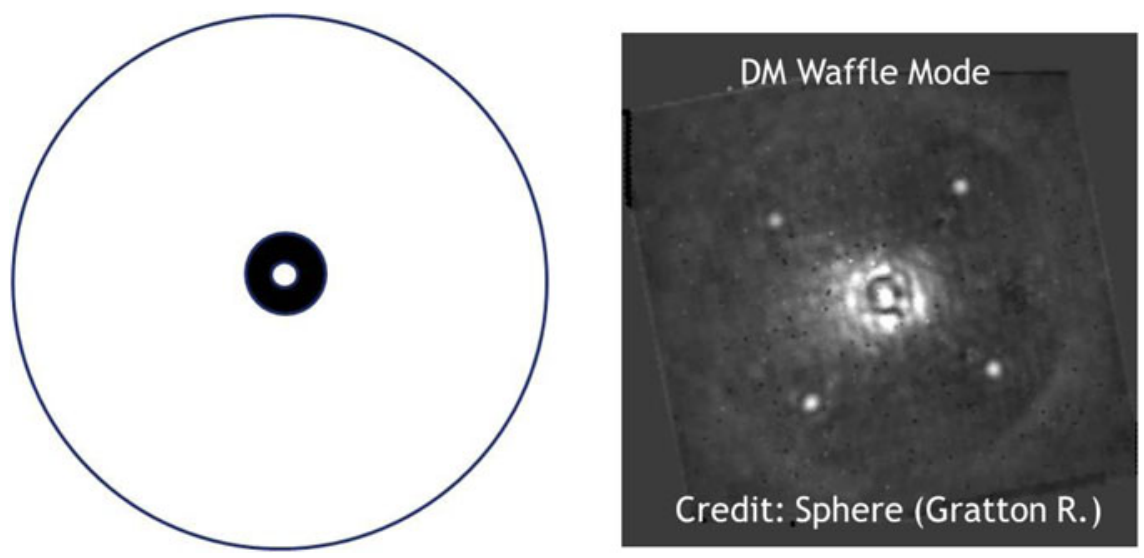

Fig. 6. On the left the mask with a central semi-transparent dot, on the right the four spots created by an artificial introduction of a waffle mode on the DM shape.

in mask positioning which can cause system performance to drop quickly. One way to track the flexures, occurring inside the instrument itself, is to follow the PSF movements during the exposure.

We have considered two ways to achieve this task. The first is the use of an occulting mask in the focal plane with a semi-transparent circle in the centre (see Fig. 6 left side); this will create a spot on the detector focal plane, allowing tracking misalignments occurring inside the instrument (i.e. between the occulting mask and the detector). This technique is suitable for coronagraphs using an occulting mask on the focal plane (Lyot), not for other types (e.g. vortex).

The second consists of creating four artificial spots on the focal plane (outside the science FoV) by introducing a waffle mode in the deformable mirror (DM): the spot movement is therefore retrievable (see Fig. 6 right side) and the telescope pointing system will compensate the PSF movements.

We note that these two methods allow for tracking the PSF movements inside the instruments, while we cannot track flexures of the overall SHARK system or of the two optical components (the dichroic and the folding mirror) located in the non-common path just before the cryogenic tank. This task is normally achieved by adding a dedicated sensor, which is checking the tip-tilt of the spot in the input focal plane by picking up a small amount (few per cent) of light through a beam splitter. In the case of SHARK NIR, we discarded this possibility for a few reasons:

- We mentioned already that the system is very compact and close to the AO WFS, and thus the non-common path aberrations are kept small by design.

- The Lyot coronagraph concept (which is the baseline design) allows for quite large shifts of the spot on the entrance focal plane without experiencing a strong decrease in the contrast. This means that the required stability of the PSF onto the occulting mask focal plane is quite low and can possibly be guaranteed by stiff mechanics, especially for a compact and light instrument as SHARK is foreseen to be.

Additionally, the initial idea of keeping the complexity of the instrument as low as possible and the limited and well-defined

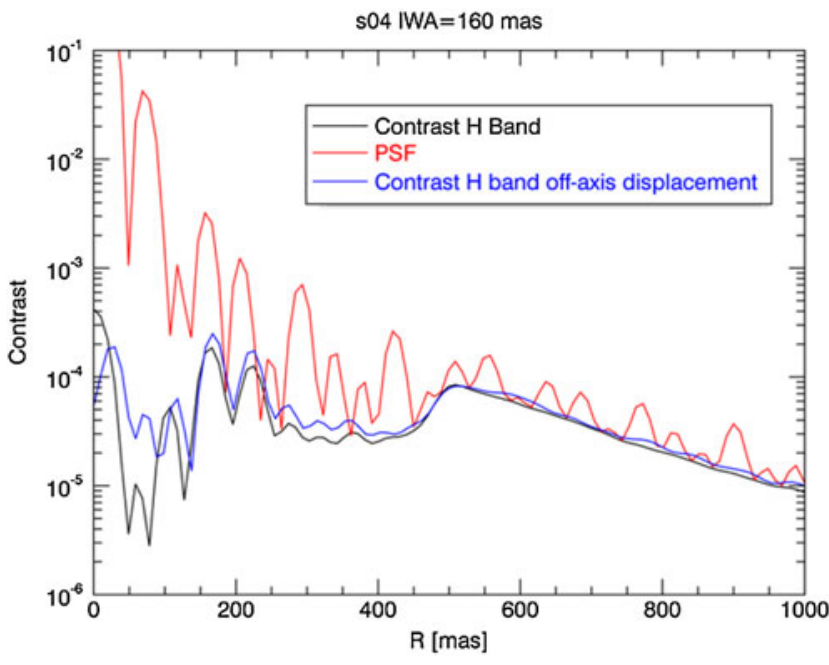

Fig. 7. The effect of a displacement of the pupil mask of $0.1 \mathrm{~mm}$ on system contrast.

available volume at the LBTI location selected for SHARK have also pushed in this direction. Nevertheless, during the final design phase, we will carefully evaluate the tolerance to displacements of the focal plane masks (similarly to what we have done for the pupil plane masks, see Fig. 7) and we will evaluate the flexures of the SHARK mechanical structure and of the components in the non-common path through more detailed finite-element analysis of the mechanical structure.

It has anyhow to be noted that, between two different scientific exposures, the image of the star will be properly centred on the focal plane mask through a dedicated procedure, and thus we will have to take into account only displacements occurring during a single scientific exposure. Preliminary estimates of the flexures and thermal effects during $1 \mathrm{~h}$ of exposure in the worse telescope position $\left(15^{\circ}\right.$ of altitude change during $1 \mathrm{~h}$ ) are causing displacements between the star and the focal plane mask of the order of $20 \mu \mathrm{m}$, which should cause a negligible decrease in 
contrast in the case of the Lyot mask, being the latter a quite wide occulting mask (normally a few $\lambda / D$ ).

\section{Tracking of the pupil movements}

In the current design, the pupil movements during a single exposure are not retrievable (but minimizing them by design). In order to test the effects of pupil movement, we have assumed flexures that cause a shift of $0.1 \mathrm{~mm}$ of the pupil mask during the exposure (very conservative case). Using a simulation code, we have then applied such a displacement, and compared the performance (in terms of contrast) with and without displacement (see Fig. 7). There is a small contrast reduction, which we consider acceptable at this stage.

Pupil rotations might be dealt with in two ways (both of them not suitable for tracking during a single exposure, but only between two of them):

- Add a pupil plane mask with 'slightly' oversized spiders, causing a loss in throughput but being less sensitive to small mismatches between the real system geometry and the pupil plane mask.

- Use the huge ASM focus adjustment to defocus the image and adjust the rotator angle accordingly.

\section{Minimization and characterization of the non-common path aberrations}

The system minimizes by design the non-common path aberrations, by making the two channels light, compact and positioned very close to the WFS.

Direct measurements made with PISCES have shown a remarkably negligible amount of non-common path aberrations. Of course, they can in any case be characterized during the daytime using the dedicated calibration source of LBTI.

Additionally, the FLAO group is currently testing an algorithm for the compensation of the pyramid variable gain with online measurement of the WFS sensitivity, which may track the non-common path aberration changes in almost real-time.

\section{Rejection of the quasi-static speckle}

Quasi-static speckles are one of the major sources of noiselimiting high-contrast imaging. Instrumental speckles (due mostly to optical quality and misalignment errors) average to form a fixed pattern, which can be calibrated to a certain extent, but their temporal evolution, limits this possibility (for example, due to mechanical and thermal deformation during the exposure).

ADI, utilized by most of the Coronagraphs, is a technique to minimize this effect (see Ren et al. 2012). The instrument acquires a sequence of images, normally with the telescope close to the Meridian, whereas the instrument field derotator is off. This keeps the instrument and telescope optics aligned and allows the FoV to rotate with respect to the instrument. For each image, a reference PSF is constructed from other appropriately selected images of the same sequence, and subtracted to remove quasi-static PSF structures. All residual images are then rotated (to align the field) and are combined.

It is also worth considering the technique of principle component analysis, an algorithm complementing ADI for increasing the contrast achievable next to a bright star (see Meshkat et al. 2014).

\section{NIR channel: precision of the motors positioning}

Some of the motorized axes require a positioning precision of the order of $1 \mu \mathrm{m}$, which is quite demanding using stepper motors in the cold, also considering the gear plays. There are several mechanical tricks to maximize the positioning precision, but we strongly suggest prototyping the more demanding axis in terms of positioning precision (the focal plane wheel), and testing in the same Cryogenic conditions as the final system.

\section{VIS channel additional issues}

The VIS channel does not have very critical issues, but the following are the most demanding aspects in the project:

- The compactness of the layout may need the development of custom opto-mechanical devices;

- The ADC optical design is challenging as it needs to ensure full band (600-950 nm) correction for the coronagraphic IFU and direct imaging mode;

- IFU fibre bundle will use small core fibres that are fragile and hard to assemble;

- Data throughputs of $250 \mathrm{MB} \mathrm{s}^{-1}$ are typical when imaging at the high frame rate, SSD devices and fast computers are mandatory. Data storage and transfer are also demanding.

\section{Conclusions}

SHARK is a simple high-contrast imager, fully exploiting the XAO provided by the pyramid-based LBT AO systems. Even if no particularly innovative concepts have been introduced in the coronagraph optical design, the instrument will be fed by an outstanding XAO system (a combination of the Pyramid WFS with a high-performing Adaptive Secondary Mirror), which is, to our knowledge, the best-performing singleconjugated AO system. It has clear opportunities to achieve primary science goals with performance, in theory, comparable with those of SPHERE and GPI and probably superior to existing facilities in the northern hemisphere, gaining an order of magnitude in contrast in the critical range of separations between 0.1 and 0.6 arcsec. Given the characteristics exploited on FLAO with respect to those of the AO systems of both SPHERE and GPI, we expect 1-2 magnitude fainter targets to be reachable (above all if the WFS upgrade to L3-CCD goes ahead), with obvious impact on the science (and on the number of targets) that might be obtainable with SHARK.

Considering the IR case, this fact would allow the observation of many more nearby small mass stars and Solar-type stars in nearby star-forming regions (e.g. Taurus at $140 \mathrm{pc}$ ). SHARK would allow the LBT to study the earliest evolution of giant planets and their link with circumstellar discs, then providing specific clues to the formation mechanism. Detailed simulations show that, with a properly selected target sample, we will be able to discover about 20-30 planets over about 50 observing nights, spread over a few years. Such a survey will complement data from SPHERE and GPI, and will 
also complement the LBT LEECH (LBTI Exozodi Exoplanet Common Hunt survey, see Skemer et al. (2014)) survey searching for planets in L - M bands, started in 2013, allowing LBT to have a leader position in this field.

In the visible domain instead, having one of the few instruments mounted on an $8 \mathrm{~m}$ class telescope obtaining good correction in this wavelength range, SHARK offers a variety of new cases that might be explored, the most attractive (and challenging) of which might be the observation of exceptionally bright exoplanets orbiting around nearby stars. In this field, SHARK will be a pathfinder of the future generation of extreme large telescopes aiming to get as close as possible to detect reflected light planets.

\section{REFERENCES}

Beuzit, J.-L. et al. (2010). Direct detection of giant extrasolar planets with SPHERE on the VLT. ASP Proc. 430, 231.

Carlotti, A., Kasdin, N.J., Vanderbei, R.J. \& Delorme, J-R. (2012). Optimized shaped pupil masks for pupil with obscuration. Proc. SPIE 8442, 54.

Costa, J.B., Feldt, M., Wagner, K., Bizenberger, P., Hippler, S., Baumeister, H., Stumpf, M., Ragazzoni, R., Esposito, S. \& Henning, T. (2004). Status report of PYRAMIR: a near-infrared pyramid wavefront sensor for ALFA. Proc. SPIE 5490, 1189.

Close, L.M. et al. (2012a). High-resolution images of orbital motion in the orion trapezium cluster with the LBT AO system. Astrophys. J. 749, 280.

Close, L.M. et al. (2012b). First closed-loop visible AO test results for the advanced adaptive secondary AO system for the Magellan Telescope: MagAO's performance and status. Proc. SPIE 8447, 0X.

Close, L.M., Follette, K.B., Males, J.R., Puglisi, A., Xompero, M., Apai, D., Najita, J., Weinberger, A.J., Morzinski, K., Rodigas, T.J., Hinz, P., Bailey, V. \& Briguglio, R. (2014). Discovery of H-alpha emission from the close companion inside the gap of transitional disk HD 142527. ApJ 781, L30-L34.

Codona, J.L. \& Angel, R. (2004). Imaging extrasolar planets by Stellar Halo suppression in separately corrected color bands. Astrophys. J. 604, L117-L120.

Currie, T., Guyon, O., Martinache, F., Clergeon, C., McElwain, M., Thalmann, C., Jovanovic, N., Singh, G. \& Kudo, T. (2013). The Subaru Coronagraphic Extreme Adaptive Optics Imager: first results and on-sky performance. Victoria Conf. Proc. 1307, 4093.

Dekany, R. et al. (2013a). PALM-3000: exoplanet adaptive optics for the $5 \mathrm{~m}$ Hale telescope. Astrophys. J. 776, 130.

Dekany, R. et al. (2013b). First exoplanet and disk results with the PALM-3000 adaptive optics system. In AO4ELT3 Conf. Proc. 52.

Esposito, S. \& Riccardi, A. (2001). Pyramid Wavefront Sensor behavior in partial correction Adaptive Optic systems. Astron. Astrophys. 369, L9.

Esposito, S. et al. (2010). First light AO (FLAO) system for LBT: final integration, acceptance test in Europe, and preliminary on-sky commissioning results. Proc. SPIE 7736, 773609.

Esposito, S. et al. (2011). Large Binocular Telescope Adaptive Optics System: new achievements and perspectives in adaptive optics. Proc. SPIE 8149, 814902.

Esposito, S. et al. (2012). Natural guide star adaptive optics systems at LBT: FLAO commissioning and science operations status. Proc. SPIE 8447, 84470 U.

Esposito, S. et al. (2013). LBT observations of the HR 8799 planetary system: first detection of HR8799e in H band. Astron. Astrophys. 549, A52.

Ghedina, A. et al. (2003). On sky test of the pyramid wavefront sensor. Proc. SPIE 4839, 869.
Guerri, G. et al. (2009). Apodized Lyot Coronagraph for VLT-SPHERE: Laboratory tests and performances of a first prototype in the visible. Astronomical Telescopes and Instrumentation, Marseille 2008, 2009arXiv: 0901.2429 .

Guerri, G. et al. (2011). Apodized Lyot coronagraph for SPHERE/VLT: II. Laboratory tests and performance. Exp. Astron. 30, 59-81.

Guyon, O., Martinache, F., Clergeon, C., Russell, R., Groff, T. \& Garrel, V. (2011). Wavefront control with the Subaru Coronagraphic Extreme Adaptive Optics (SCExAO) system. SPIE Proc. 8149, 894293.

Hagelin, S., Masciadri, E. \& Lascaux, F. (2011). Optical turbulence simulations at Mt Graham using the Meso-NH model. Mon. Not. R. Astron. Soc. 412, 2695.

Hinz, P.M., Angel, J.R.P., McCarthy, D.W., Jr., Hoffman, W.F. \& Peng, C.Y. (2003). The large binocular telescope interferometer. Proc. SPIE 4838, 108.

Lyot, B. (1939). Mon. Not. R. Astron. Soc. 99, 580.

Macintosh, B.A. et al. (2008). The Gemini Planet Imager: from science to design to construction. Proc. SPIE 7015, 31.

Males, J.R. et al. (in preparation). High contrast imaging of an exoplanet with the Magellan VisAO Camera. IAU Proc. 46.

Mandel, H. et al. (2007). LUCIFER: a NIR Spectrograph and Imager for the LBT. Astron. Nachr. 328(7), 626.

Martinez, P., Boccaletti, A., Kasper, M., Cavarroc, C., Yaitskova, N., Fusco, T. \& Vérinaud, C. (2008). Comparison of coronagraphs for high-contrast imaging in the context of extremely large telescopes. Astron. Astronphys. 492, 289.

Masciadri, E., Stoesz, J., Hagelin, S. \& Lascaux, F. (2010). Optical turbulence vertical distribution with standard and high resolution at $\mathrm{Mt}$ Graham. Mon. Not. R. Astron. Soc. 404, 144.

Mawet, D., Riaud, P., Absil, O. \& Surdej, J. (2005). Annular groove phase mask coronagraph. Astrophys. J. 633, 1191.

Meshkat, T., Kenworthy, M.A., Quanz, S.P. \& Amara, A. (2014). Optimized principal component analysis on coronagraphic images of the Fomalhaut system. Astrophys. J. 780, 17.

Quiròs-Pacheco, F., Briguglio, R., Pinna, E., Puglisi, A., Riccardi, A. \& Esposito, S. (2011). FLAO\#1 Commissioning Report. LBT-ADOPT Technical Report nr. 485f032, Version a, Date 05-12-2011

Ragazzoni, R. (1996). Pupil plane wavefront sensing with an oscillating prism. JMOp 43, 289.

Ragazzoni, R. \& Farinato, J. (1999). Sensitivity of a pyramidic Wave Front sensor in closed loop Adaptive Optics. Astron. Astrophys. 350, L23.

Ren, D., Dou, J., Zhang, X. \& Zhu, Y. (2012). Speckle noise subtraction and suppression with adaptive optics coronagraphic imaging. Astrophys. $J$. 753, 99.

Riccardi, A. et al. (2010). The adaptive secondary mirror for the Large Binocular Telescope: optical acceptance test and preliminary on-sky commissioning results. Proc. SPIE 7736, 79.

Rodigas, T.J. et al. (2012). The grey needle: large grains in the HD 15115 debris disk from LBT/PISCES/Ks and LBTI/LMIRcam/L' adaptive optics imaging. Astrophys. J. 752, 57.

Salinari, P., Del Vecchio, C. \& Biliotti, V. (1994). A study of an adaptive secondary mirror. In ESO Proc. Conf. on Active and Adaptive Optics, 247.

Skemer, A.J. et al. (2014). High contrast imaging at the LBT: the LEECH exoplanet imaging survey. SPIE 9148, 914820.

Thomas, S.J. et al. (2011). Integration and test of the Gemini Planet Imager. Proc. SPIE 8149, 814903.

Vérinaud, C., Le Louarn, M., Korkiakoski, V. \& Carbillet, M. (2005). Adaptive optics for high-contrast imaging: pyramid sensor versus spatially filtered Shack-Hartmann sensor. Mon. Not. R. Astron. Soc. 357, L26.

Wilson, J.C., Hinz, P.M., Skrutskie, M.F., Jones, T., Solheid, E., Leisenring, J., Garnavich, P., Kenworthy, M., Nelson, M.J. \& Woodward, C.E. (2008). LMIRcam: an L/M-band imager for the LBT combined focus. Proc. SPIE 7013, 101. 